\title{
Horn type and horn length genes map to the same chromosomal region in Soay sheep
}

\author{
SE Johnston ${ }^{1}$, D Beraldi ${ }^{2}$, AF McRae ${ }^{3}$, JM Pemberton ${ }^{2}$ and J Slate ${ }^{1}$ \\ ${ }^{1}$ Department of Animal and Plant Sciences, University of Sheffield, Sheffield, UK; ${ }^{2}$ Institute of Evolutionary Biology, School of Biological \\ Sciences, University of Edinburgh, Edinburgh, UK and ${ }^{3}$ Queensland Statistical Genetics, Queensland Institute of Medical Research, \\ Brisbane, Australia
}

\begin{abstract}
The evolution of male weaponry in animals is driven by sexual selection, which is predicted to reduce the genetic variability underlying such traits. Soay sheep have an inherited polymorphism for horn type in both sexes, with males presenting with either large, normal horns or small, deformed horns (scurs). In addition, there is additive genetic variation in horn length among males with normal horns. Given that scurred males cannot win conflicts with normal-horned males, it is unusual that genes conferring scurs should persist in the population. Identifying the genetic basis of these traits should help us in understanding their evolution. We developed microsatellite markers in a targeted region of the Soay sheep genome and refined the location of the
\end{abstract}

Horns locus $(\mathrm{Ho})$ to a $\sim 7.4 \mathrm{cM}$ interval on chromosome 10 $(\mathrm{LOD}=8.78)$. We then located quantitative trait loci spanning a $34 \mathrm{cM}$ interval with a peak centred close to $\mathrm{Ho}$, which explained the majority of the genetic variation for horn length and base circumference in normal-horned males $(L O D=2.51$ and $L O D=1.04$, respectively). Therefore, the genetic variation in both horn type and horn length is attributable to the same chromosomal region. Understanding the maintenance of horn type and length variation will require an investigation of selection on genotypes that (co)determine both traits.

Heredity (2010) 104, 196-205; doi:10.1038/hdy.2009.109; published online 19 August 2009

Keywords: quantitative trait locus mapping; horn morphology; natural population; genetic correlation; sexual selection; trade-off

\section{Introduction}

Natural and sexual selection in the wild are often directional, and therefore alleles conferring unfavourable phenotypes are expected to be removed from the population. This in turn should lead to a reduction in the genetic variability underlying the trait and to a permanent change in its distribution (Falconer and Mackay, 1996). However, substantial genetic variation is often maintained despite apparent directional selection (Fisher, 1958; Houle, 1992). This is particularly evident for wild populations, in which rapid and unpredictable environmental change can lead to a shift in the optimum phenotype, or more genetic variance is exposed due to changes in the genotype by environment interactions (Merilä and Sheldon, 1999; Kruuk, 2004; Kruuk et al., 2008). The extension of traditional quantitative genetic methods to account for this stochasticity in the wild is continually improving our understanding of how freeliving populations respond to selection (Kruuk et al., 2008) and is reflected in several informative studies of microevolution in wild vertebrates, in particular, in ruminants (Kruuk et al., 2000, 2002; Coltman et al., 2005; Wilson et al., 2005), birds (Garant et al., 2005;

Correspondence: SE Johnston, Department of Animal and Plant Sciences, Alfred Denny Building, University of Sheffield, Western Bank, Sheffield, S10 2TN, UK

E-mail:S.Johnston@shef.ac.uk

Received 5 May 2009; revised 1 July 2009; accepted 20 July 2009; published online 19 August 2009
Nussey et al., 2005; Charmantier et al., 2006; Jensen et al., 2008), rodents (Réale et al., 2003) and salmonids (Thériault et al., 2007). Although these studies successfully quantify the proportion of variation in life history and morphology phenotypes attributed to environmental differences and additive genetic effects, pinpointing the source of genetic variability often remains elusive. This is because traditional methods alone give little indication of the underlying genetic architecture of a focal trait, that is, the number of genes involved, their relative effects, mode of inheritance and/or their actions and interactions with additional characters and environmental conditions. One key step to resolve this issue is to identify the genetic basis of both discrete and quantitative characters within wild populations, as this will provide the foundation required to investigate the role of particular genes and genomic regions in maintaining genetic variation.

The recent expansion of genomic resources and the improved efficiency of sequencing and genotyping technology has made the elucidation of genetic architecture in pedigreed wild populations much more tractable (Ellegren and Sheldon, 2008). This is reflected in recent research using gene mapping and quantitative trait locus (QTL) methodology to identify genomic regions responsible for quantitative and simple Mendelian trait variation (Slate et al., 2002; Gratten et al., 2007; Beraldi et al., 2006, 2007b). Recently, Gratten et al. (2008) showed how identification of the underlying genotype of coat colour variation in the Soay sheep can be used 
to make inference about microevolutionary processes, which would have been cryptic when examining variation at the level of phenotype. Therefore, gene mapping does not simply explain the underlying genetic basis of a trait, but it can also be used to make inference about causes of microevolutionary change (or stasis).

For species in which males compete for the opportunity to mate, the evolution of exaggerated male weaponry may be driven by sexual selection, because of the advantage it confers in intra-sexual competition (Andersson, 1994; Pomiankowski and Møller, 1995). The feral Soay sheep (Ovis aries) of the island of Hirta (St Kilda, Scotland) has a promiscuous mating system, with fierce competition between males for access to oestrous females during the rutting season (Coltman et al., 1999). The majority of males possess large horns, which are used as a weapon in intra-male conflict. However, the Soay sheep are unusual in that they have an inherited polymorphism for horn type in both sexes, with substantial quantitative variation within each horn type. Males have two distinct horn phenotypes, namely large, normal horns (87\% of all records) and scurred horns (vestigial and deformed horns, 13\%; Figure 1). Females typically have smaller horns and have three distinct phenotypes, namely normal $(32 \%)$, scurred $(29 \%)$ and polled (complete absence of horns, 39\%). Pedigree data suggest that the polymorphism could be controlled by a single autosomal locus, Horns $(\mathrm{Ho})$, with three alleles, normal-horned $\left(\mathrm{Ho}^{+}\right)$, sex-limited $\left(\mathrm{Ho}^{\mathrm{L}}\right)$ and polled $\left(\mathrm{Ho}^{\mathrm{P}}\right)$ (Coltman and Pemberton, 2004). This model is identical to that originally proposed for Merino sheep (Dolling, 1961), and is characterized by differences in dominance and expression of particular alleles between the sexes, making the inference of the individual genotype at $\mathrm{Ho}$ difficult (Table 1). Quantitative variation within each horn type, such as horn length in normal-horned males, also has a heritable component (Preston et al., 2003; Robinson et al., 2008).

The Soay sheep population has been the subject of a long-term project addressing a number of questions relating to their evolution and ecology (see Clutton-Brock and Pemberton (2004b) for more information). Several studies have offered explanations as to why the genetic variation in both horn type and horn morphology within normal horns is maintained in this population, by examining selection on both horn type and horn length. Among normal-horned males, animals with longer horns sire more offspring because of the advantage their larger horns confer in intra-male conflict, suggesting strong sexual selection on horn length (Preston et al., 2003;

Table 1 Phenotypic distribution and the underlying genotypes of horn type in genotyped individuals in the Soay mapping pedigree in which horn phenotype is known

\begin{tabular}{lclc}
\hline Sex & Horn type & Genotype & Frequency \\
\hline $\begin{array}{l}\text { Males } \\
n=273\end{array}$ & Normal & $\mathrm{Ho}^{+} \mathrm{Ho}^{+}$ & 0.89 \\
& & $\mathrm{Ho}^{+} \mathrm{Ho}^{\mathrm{L}}$ & \\
& $\mathrm{Ho}^{+} \mathrm{Ho}^{\mathrm{P}}$ & \\
& $\mathrm{Ho}^{\mathrm{L}} \mathrm{Ho}^{\mathrm{L}}$ & \\
& $\mathrm{Ho}^{\mathrm{L}} \mathrm{Ho}^{\mathrm{P}}$ & \\
& & $\mathrm{Ho}^{\mathrm{P}} \mathrm{Ho}^{\mathrm{P}}$ & 0.11 \\
$\begin{array}{l}\text { Females } \\
n=286\end{array}$ & Scurred & $\mathrm{Ho}^{+} \mathrm{Ho}^{+}$ & 0.381 \\
& Normal & $\mathrm{Ho}^{+} \mathrm{Ho}^{\mathrm{L}}$ & \\
& Scurred & $\mathrm{Ho}^{+} \mathrm{Ho}^{\mathrm{P}}$ & 0.248 \\
& Polled & $\mathrm{Ho}^{\mathrm{L}} \mathrm{Ho}^{\mathrm{L}}$ & \\
& $\mathrm{Ho}^{\mathrm{L}} \mathrm{Ho}^{\mathrm{P}}$ & 0.371 \\
& $\mathrm{Ho}^{\mathrm{P}} \mathrm{Ho}^{\mathrm{P}}$ & \\
\hline
\end{tabular}

Frequencies differ slightly from population-wide frequencies described in the section 'Introduction.'

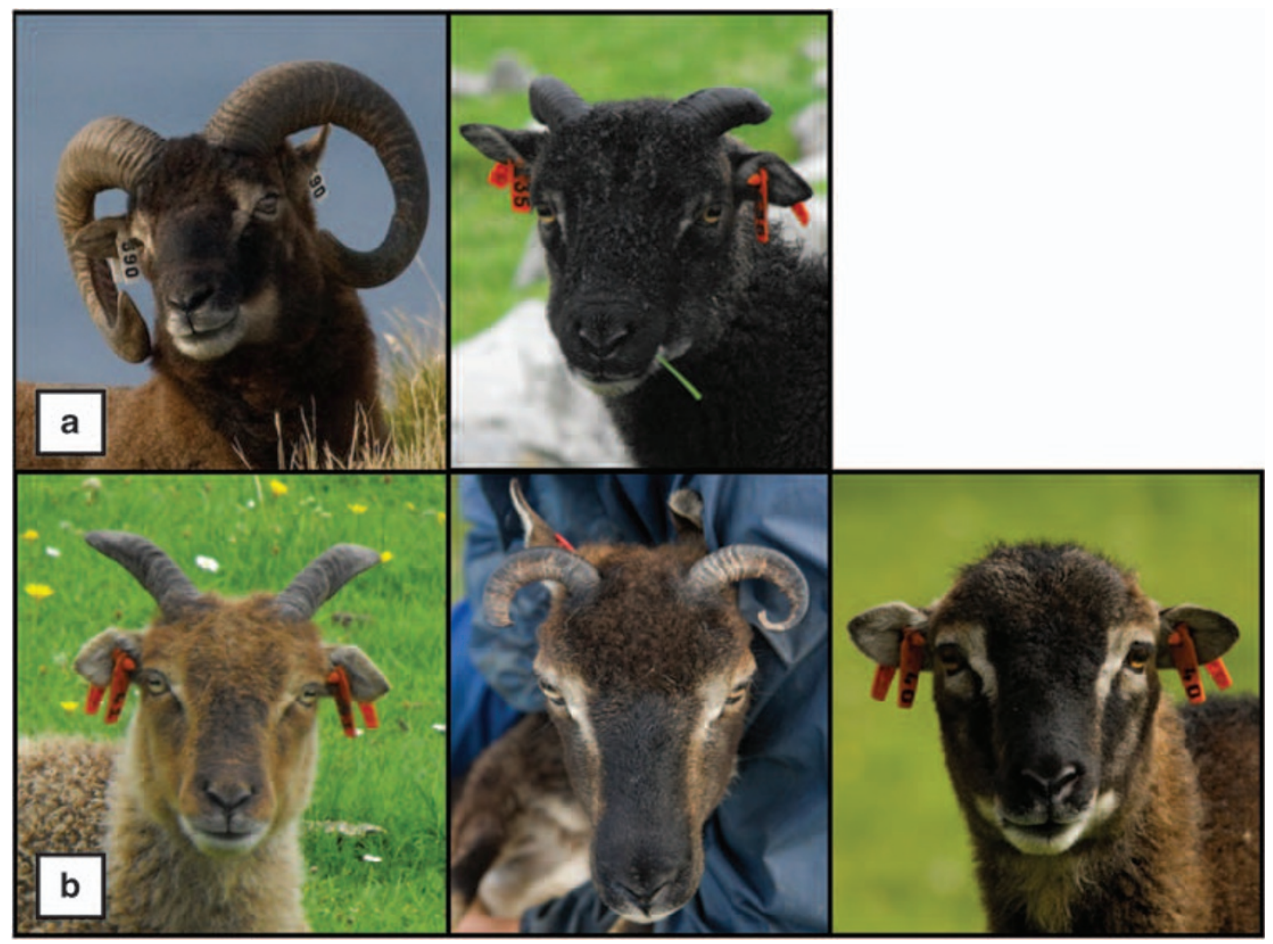

Figure 1 Soay sheep horn types in adult males and females in the Village Bay population. Males (a): normal (left) and scurred (right); females (b): normal-horned (left), scurred (centre) and polled (right) (Photographs taken by Arpat Ozgul and Susan Johnston). 
Robinson et al., 2006). Normal-horned males have the greatest reproductive success in a given year, but have reduced longevity and poorer over-winter survival when compared to scurred males (Robinson et al., 2006). Furthermore, larger normal horns are costly to produce and may only be advantageous if males experience favourable environmental conditions in the first year of life (Robinson et al., 2008). In female Soays, scurred females conceive more offspring, have greater weaning rates and over-winter survival when compared to normal and polled females (Clutton-Brock et al., 1997; Robinson et al., 2006). This evidence suggests that the horn-type polymorphism is maintained by antagonistic selection between the sexes, whereas variation in horn length is maintained by its effect on male fitness changing with environmental conditions.

The development of a linkage map covering 90\% of the Soay sheep genome (Beraldi et al., 2006) has proved indispensable in further investigations of the genetic basis of several quantitative and discrete traits in this population (Beraldi et al., 2006, 2007a,b; Gratten et al., 2007). So far, a coat colour polymorphism has been fine mapped to the TYRP1 gene on chromosome 2 (Gratten et al., 2007), and scans for QTLs underlying morphological traits and parasite resistance have had some success, including the discovery of a genome-wide significant QTL for jaw length on chromosome 11 (Beraldi et al., 2007a,b). As yet, it is unknown whether both horn type and horn length variation (among normal-horned males) are genetically correlated, that is, whether they share a common underlying set of genes (or genomic regions). Given the complex nature of selection on these traits, investigations of the evolution of horns in the population will benefit from being able to distinguish sheep with the same horn type, but with different underlying genotypes, as well as the identification of genomic regions that affect variation in horn length. The Ho locus has been mapped to a $\sim 16 \mathrm{cM}$ region of chromosome 10 in separate mapping experiments in both domestic and Soay sheep (Montgomery et al., 1996; Beraldi et al., 2006), but the gene responsible has yet to be characterised.

In this paper, we map Ho with improved resolution by developing new molecular markers in a targeted region of the Soay sheep genome using online genomic sequence information obtained from related domestic organisms. We also report a QTL analysis of horn morphology, examining horn length and base circumference in normal-horned males, and present evidence that both horn type and horn length have a shared genetic basis. Finally, we discuss how this study provides a foundation for future work using the underlying genotype to understand the evolution of horn type and morphology in this and in other populations.

\section{Materials and methods}

\section{Study population}

The Soay sheep of St Kilda (Scotland, UK; $57^{\circ} 49^{\prime}$ N, $8^{\circ} 34^{\prime}$ $\mathrm{W})$ are a primitive breed likely to be the survivors of the earliest domestic sheep that spread across Europe in the Bronze Age (Clutton-Brock and Pemberton, 2004a). The study population in Village Bay, Hirta, was established in 1932 with the introduction of 107 sheep from the neighbouring island of Soay, and has existed unmanaged ever since. The Village Bay population has been studied on an individual basis since 1985 (CluttonBrock et al., 2004). Each spring, $>95 \%$ of lambs are caught, ear-tagged and sampled for genetic analysis. No predators are present on St Kilda.

\section{Horn phenotype data set}

Phenotypic records of sheep in the mapping pedigree were retrieved from the Soay Sheep Project Database, which contains data on more than 6800 sheep. Normal and scurred horns grow cumulatively throughout the lifetime of the sheep. Horn type and dimensions are recorded annually during a 2-week period in August (in which $49-67 \%$ of the study population are captured), during the rut in November and December and/or after death. Horn length was measured as the length (in millimetre) from the base of the horn, along the outer curvature of the spiral to the tip. Horn base circumference was measured as the circumference (in millimetre) around the base of the horn at the closest point to the skull.

\section{Mapping pedigree and genome-wide linkage map}

The Soay sheep mating system is promiscuous, meaning that few full-sibs occur in the population. Maternal links were assigned through field observations, whereas paternal links were inferred through molecular analysis (Overall et al., 2005). The whole Soay sheep pedigree contains more than 3900 animals, from which a pedigree of 882 were selected as a mapping panel, comprising all sibships with $\geqslant 10$ offspring and their common parents (Beraldi et al., 2006); 588 animals within this pedigree were genotyped, with the remaining animals serving to improve estimates of kinship and identity-by-descent coefficients used in the variance components analysis. A genome-wide linkage map was constructed by Beraldi et al. (2006) and covered $\sim 90 \%$ of the genome with an average intra-marker interval of $\sim 15 \mathrm{cM}$. Further information on the mapping pedigree selection criteria, map characteristics and technical procedures are available in the study by Beraldi et al. (2006).

\section{Developing microsatellite markers around the Ho locus}

Previous research has mapped $H o$ to a $\sim 16 \mathrm{cM}$ region on chromosome 10, which has conserved synteny with cattle chromosome 12 (Montgomery et al., 1996; Beraldi et al., 2006). The previous linkage map of Soay sheep chromosome 10 consisted of nine markers, of which only one (AGLA226) was associated with horn type $(\mathrm{LOD}=6.1$; Beraldi et al. (2006)). Therefore, a higher resolution mapping of $H o$ required an increase in marker density in a targeted region of chromosome 10, corresponding to a $\sim 25-\mathrm{Mb}$ region on bovine chromosome 12 . We screened all available domestic sheep DNA sequences ( $n=569364$, NCBI Genbank Database, accessed November 2006) for microsatellite repeat motifs using modified Sputnik II software (http://wheat.pw.usda. gov/ITMI/EST-SSR/LaRota/) with the following parameters: repeat motif $=$ di- or tetra-nucleotide, minimum length of repeat sequence $=30$ bases and repeat purity $\geqslant 95 \%$, of which 5399 sequences met this criteria. These sequences were aligned to the entire bovine genome (version Btau2.0.14, available from ftp://ftp.ensembl. 
org/, accessed November 2006) using the blastn command in BLAST (v 2.2.15, Altschul et al., 1990), with the following parameters: word size $=11$ and expectation value $=10^{-20}$. We selected 17 sequences, which aligned solely to a single region on the homologous region of cattle chromosome 12 as candidate loci (named OarSEJ ${ }_{n}$, see Supplementary Table S1). Specific PCR primers for each microsatellite were designed using Primer3 (Rozen and Skaletsky, 2000).

\section{DNA extraction and microsatellite genotyping}

Genomic DNA was extracted from blood samples or from ear-punch tissue using DNeasy Tissue Kit (QIAGEN, Crawley, UK). OarSEJ ${ }_{n}$ loci were initially tested in six sheep, to verify positive amplification and to optimize the annealing temperature and $\mathrm{MgCl}_{2}$ concentration. Successful loci were then typed in 14-34 sheep to test for polymorphism and to size products for multiplex PCR. Fluorescent forward primers were synthesized by Operon Biotechnologies $\mathrm{GmbH}$ (Cologne, Germany) (6FAM and 5HEX labels) and by Applied Biosystems (Warrington, UK) (NED label). Three reverse primers (OarSEJ07, OarSEJ10 and OarSEJ16) were pigtailed at the 5 -end with the sequence GTTTCTT to improve scoring reliability (Brownstein et al., 1996). PCR amplifications for individual loci were performed in $10 \mu \mathrm{l}$ volume containing $1 \mu \mathrm{l} 10 \times \mathrm{NH}_{4}$ buffer, $0.2 \mathrm{mM}$ dNTPs, 2.5$4.0 \mathrm{mM} \mathrm{MgCl} 2,1 \mathrm{U}$ BioTaq and $10 \mathrm{ng}$ genomic DNA. Annealing temperatures were adjusted between 59 and $64{ }^{\circ} \mathrm{C}$ to achieve optimal reaction quality (Supplementary Table S1). Amplifications were performed using the following conditions: initial denaturation at $94^{\circ} \mathrm{C}$ for $3 \mathrm{~min}, 30$ cycles consisting of denaturation at $94^{\circ} \mathrm{C}$ for $30 \mathrm{~s}$, annealing at $55-65^{\circ} \mathrm{C}$ for $30 \mathrm{~s}$, and extension at $72{ }^{\circ} \mathrm{C}$ for $30 \mathrm{~s}$ and a final extension at $72{ }^{\circ} \mathrm{C}$ for $5 \mathrm{~min}$. Multiplex reactions were performed in three groups, each with between four and six primer pairs arranged for product size and primer label, using the Qiagen Multiplex Kit in $2 \mu \mathrm{l}$ reactions containing $1 \mu \mathrm{l}$ of the Multiplex Kit, $0.2 \mu \mathrm{M}$ of each primer pair, $\mathrm{dH}_{2} \mathrm{O}$ and $5 \mathrm{ng}$ air-dried DNA. The PCR conditions followed the manufacturer's protocol with an annealing temperature of $61^{\circ} \mathrm{C}$. PCR product lengths were analysed on an ABI 3730 DNA Analyzer, and genotypes were scored using Genemapper v3.7 (Applied Biosystems). Pedigree mismatches were tested using PEDSTATS (Wigginton and Abecasis, 2005) and PedCheck (O'Connell and Weeks, 1998), and were resolved by rechecking parentage, genotypes, chromatograms or by scoring problematic animals as untyped. Genotyping error rate and estimates of null allele frequency were calculated using CERVUS 3.0 (Kalinowski et al., 2007).

\section{Chromosome 10 linkage map}

The linkage map was constructed using CRI-MAP v2.4 (Green et al., 1990), which determined marker order, map distances, two-point LOD scores and the number of informative meioses at each locus. The Soay mapping pedigree is complex in that it contains numerous inbreeding loops and every sheep is related. Therefore, to increase computational efficiency, the pedigree was simplified into 29 discrete three-generation families using the CRIGEN utility for CRI-MAP (Liu and Grosz, 2006). This mapping analysis combined marker informa- tion obtained from $\mathrm{OarSEJ}_{n}$ markers and those mapped to chromosome 10 by Beraldi et al. (2006). Polymorphic $\mathrm{OarSEJ}_{n}$ markers were tested for linkage to all markers on the Soay Genome Linkage Map using the twopoint function, to ensure exclusive linkage to loci on chromosome 10. Multipoint linkage analysis was carried out with all markers known to be on chromosome 10 using the build function, which uses the sequential incorporation of loci and determines marker order and intermarker distances. The log10 likelihood of the initial marker order from the build function was compared with that of alternative orders using the flips function. Individual recombination events and recombination locations were examined using the chrompic function. The chrompic function identified animals with dubious double recombination events occurring between closely linked loci, which were reassessed and removed if necessary.

\section{Mapping the Ho locus}

The Ho locus was mapped using a parametric multipoint linkage analysis in LINKAGE (Terwilliger and Ott, 1994). The mapping panel was split into 39 unlinked families (Beraldi et al., 2006), with each sheep assigned to one of five liability classes on the basis of horn phenotype and sex (see Table 1), and a sixth class for animals with unclassified horn phenotype. The horn allele frequencies were specified as $0.441,0.170$ and 0.389 for $\mathrm{Ho}^{+}, \mathrm{Ho}^{\mathrm{L}}$ and $H o^{\mathrm{P}}$, respectively (as stated in Coltman and Pemberton, 2004), and the underlying genotypes in each class were assumed to have complete penetrance. Genome-wide evidence of linkage was accepted with a LOD threshold of 3.31, and a 95\% confidence interval for gene location was determined using a 1-LOD decrease (see section 'QTL mapping and estimation of QTL effect').

\section{Locating QTL for horn dimensions in normal-horned males}

The mapping analyses described in the previous section are appropriate for identifying the loci that determine traits with discrete phenotypic classes (inter-horn type variation), but not for quantitative (continuously varying) traits, such as horn length in normal-horned males (intra-horn type variation). To map QTL, we adopted (and further developed) a variance components approach (George et al., 2000; Slate, 2005).

Definitions of fixed effects: A general linear mixed model was implemented in $\mathrm{R}$ ( $\mathrm{R}$ Development Core Team, 2008) to determine the fixed effects with a significant contribution to variation in horn traits. Capture age was fitted as a factor with nine levels (ages 0 to 8 years or more), and capture period was fitted as a factor with the following four levels: January-March, April-June, July-September and October-December. Each fixed effect was added sequentially to the model for each phenotype and the significance was calculated using a Wald statistic, assuming a $\chi^{2}$ distribution with $n-1$ degrees of freedom (d.f.), where $n$ is the number of levels within the factor. Animal identity was included as a random effect to account for repeated measures. 
Estimating variance components: The null hypothesis of no segregating QTL assumes the source of additive genetic variation is many genes of small effect randomly scattered through the genome. This is represented by the 'polygenic' model. Horn morphology in normal-horned males in the mapping pedigree can be modelled as a combination of fixed and random effects using the Animal Model (Lynch and Walsh, 1998; Kruuk, 2004):

$$
y=X \beta+Z u+\varepsilon,
$$

where $y$ is a vector of phenotypic observations over all animals, $\beta$ a vector of fixed effects, $u$ a vector of random effects, $X$ and $Z$ design matrices of relating records to appropriate fixed and random effects, respectively and $\varepsilon$ a vector of residual effects. This framework can be used to partition several random effects, including the additive genetic effect. The animal model differs from a standard mixed model in that it uses pedigree information to estimate additive genetic effects, incorporating the correlations of effects between relatives. Horn measurements are often recorded at different life stages within the same animal. Therefore, the identity of the animal was fitted as a random effect to group these repeated measurements. This allowed us to quantify the variance attributed to permanent betweensheep differences, hereafter referred to as the permanent environmental effect. Finally, both birth year and capture year were fitted to account for the variation attributed to specific environmental effects associated with these years. Although there is a phenotypic correlation between horn length and body weight $\left(r_{\mathrm{P}}=0.410\right.$, s.e. $=0.047$ ), fitting body weight as a covariate did not affect the proportion of variance explained by each random effect or the significance of the QTL model. In addition, not all animals were weighed when horn measurements were taken. Therefore, we did not include body weight in our models, although models with body weight are reported in Supplementary information (Document S2).

The phenotypic variance $\left(V_{\mathrm{P}}\right)$ was calculated as the sum of all variance components, and heritability $\left(h^{2}\right)$, permanent environmental effect $\left(c^{2}\right)$, birth year effect $\left(b^{2}\right)$, capture year effect $\left(y^{2}\right)$ and the residual effect $\left(\varepsilon^{2}\right)$ were calculated as the ratio of the relevant variance component $\left(V_{\mathrm{A}}, V_{\mathrm{C}}, V_{\mathrm{B}}, V_{\mathrm{Y}}\right.$ and $V_{\varepsilon}$, respectively) to the phenotypic variance, that is, $h^{2}=V_{\mathrm{A}} / V_{\mathrm{P}}, c^{2}=V_{\mathrm{C}} / V_{\mathrm{P}}$ $b^{2}=V_{\mathrm{B}} / V_{\mathrm{P}}, y^{2}=V_{\mathrm{Y}} / V_{\mathrm{P}}$ and $\varepsilon^{2}=V_{\varepsilon} / V_{\mathrm{P}}$. We also calculated the observed phenotypic variance in the raw phenotypic data. Both capture age and capture period were included as fixed effects. Variance components were estimated using a REML (restricted maximum likelihood procedure) (Lynch and Walsh, 1998) implemented in ASReml 1.0 (Gilmour et al., 2002).

QTL mapping and estimation of QTL effect: To test for the presence of a QTL, a second-linear mixed model was generated with the same terms as those of the polygenic model, plus a QTL effect at a genomic location of interest (Slate, 2005):

$$
y=X \beta+Z u+Z q+\varepsilon,
$$

where $q$ is a vector of additive QTL effects. To map putative segregating QTL, an identity-by-descent matrix was first estimated at a given map position using pedigree relationships, marker information and map distances. Identity-by-descent matrices were estimated every $5 \mathrm{cM}$ along the whole genome map, and every $1 \mathrm{cM}$ at putative QTL regions. Identity-by-descent matrices were calculated using a Markov Chain Monte Carlo process, implemented in the program Loki (Heath, 1997). LOD scores were calculated as the difference in log likelihood between the polygenic and QTL model according to the equation,

$$
\mathrm{LOD}=\left(\mathrm{L}_{\mathrm{QTL}}-\mathrm{L}_{\text {Polygenic }}\right) / \ln (10)
$$

where $L$ is the log likelihood of the specified model. Using the formula given in Box 1 of the study by Lander and Kruglyak (1995), the significance thresholds for declaring evidence of a QTL are 3.31 for genome-wide significance (a test statistic expected to be exceeded by chance once in every 20 genome scans) and 1.88 for suggestive linkage (a false positive expected once in a single genome scan; Nyholt, 2000). These correspond closely to thresholds suggested by Lander and Kruglyak (1995) for human pedigrees, as the number of chromosomes and linkage map lengths $(\sim 3300 \mathrm{cM})$ of the Soay sheep and humans are similar. Confidence intervals for the presence of a putative QTL were defined as the map distance within a 1-LOD score decrease from the peak value, equivalent to $\sim 95 \%$ confidence (Lander and Botstein, 1989). The nominal significance of the QTL model against the polygenic model was also calculated using a likelihood ratio test (LRT):

$$
L R T=2\left(L_{\mathrm{QTL}}-L_{\text {Polygenic }}\right),
$$

where $L$ is the log likelihood of the specified model. The LRT is distributed as 50:50 mixture of a $\chi^{2}$ with 1 d.f. and a point mass of zero; therefore, the significance value is obtained by assuming a $\chi^{2}$ with 1 d.f and halving the $P$-value (Almasy and Blangero, 1998). We report nominal significance thresholds, because we wanted to test $a$ priori whether the Ho locus explained the variation in horn morphology within normal-horned males. It can be argued that applying genome-wide thresholds to this analysis is overly conservative.

We fitted QTL models with age and capture period fitted as fixed effects. The variance was partitioned into heritability, permanent environmental effect, capture year effect $\left(y^{2} ; V_{\mathrm{Y}} / V_{\mathrm{P}}\right)$, birth year effect $\left(b^{2} ; V_{\mathrm{B}} / V_{\mathrm{P}}\right)$ and QTL effect $\left(q^{2} ; V_{\mathrm{Q}} / V_{\mathrm{P}}\right)$, where the phenotypic variance $\left(V_{\mathrm{P}}\right)$ is the sum of the variance components.

Bivariate QTL models: Univariate variance component analyses of two quantitative traits can show evidence for QTL at the same location, but are unable to show whether QTL alleles cause a positive, negative or even zero localized correlation between the traits. Therefore, we carried out a bivariate QTL analysis that tested whether the Ho locus affected both horn length and horn base circumference. Random effects were fitted sequentially and tested using LRTs (see above). We compared models in which (1) both traits had polygenic variation but no QTL; (2) one trait only had a QTL at Ho (one QTL parameter estimated); (3) both traits had a QTL at $H o$, with a QTL correlation constrained to zero (two QTL parameters); (4) both traits had a QTL at Ho with a QTL correlation constrained to one (two QTL parameters); and (5) both traits had a QTL at Ho, with the sign and magnitude of the QTL correlation 
unconstrained (three QTL parameters). The correlation between two traits for a given random effect was calculated as follows:

$$
r_{i}=\operatorname{Cov}[x, y] /\left[\left(V_{i x}\right)\left(V_{i y}\right)\right]^{0.5},
$$

where $r_{i}$ is the correlation for random effect $i, x$ and $y$ the traits under comparison and $V_{i}$ the variance associated with random effect $i$.

\section{Results}

\section{Marker development}

Of the 17 candidate OarSEJ ${ }_{n}$ loci, 15 amplified successfully in the Soay sheep. Two loci (OarSEJ03 and OarSEJ13) exhibited high frequencies of parent-offspring mismatches, in which each animal was homozygous for a different allele. CERVUS estimated null allele frequencies in these markers as 0.282 and 0.106 , respectively. OarSEJ03 was rejected from further analyses; OarSEJ13 was retained in analysis by identifying animals likely to be carrying the null allele and by reclassifying the genotypes at this locus as heterozygous for both the observed allele and the null allele.

\section{Chromosome 10 linkage map}

Of the remaining markers, OarSEJ14 did not map to chromosome 10, but showed strong linkage to three markers on chromosome 23. OarSEJ05 had low polymorphism and an accurate map position could not be determined, and hence was removed from this analysis. The updated chromosome 10 linkage map was constructed with 21 microsatellite markers, incorporating $12 \mathrm{OarSEJ}_{n}$ markers and 9 markers from Beraldi et al. (2006), with a total of $\sim 10600$ genotypes. The map increased in length from 123.2 to $144.5 \mathrm{cM}$ (Kosambi's mapping function), and the $\mathrm{OarSEJ}_{n}$ markers spanned an interval of $60.5 \mathrm{cM}$. This updated map is longer because OarSEJ02 and OarSEJ06 are telomeric to all markers on the previous map, rather than due to intermarker interval inflation. Recombination fractions between adjacent loci were also used to reconstruct the map using Haldane's mapping function, to allow compatibility with downstream analyses in LINKAGE and Loki.

\section{Horn type: mapping the Ho locus}

Two-point parametric mapping in LINKAGE detected association between horn type and five markers on chromosome 10, with OarSEJ10 showing the strongest association $(\mathrm{LOD}=8.6)$; in addition, AGLA226 $(\mathrm{LOD}=6.1)$, OarSEJ13 $(\mathrm{LOD}=4.5)$, OarSEJ11 $(\mathrm{LOD}=4.1)$ and OarSEJ07 $(\mathrm{LOD}=2.5)$ were also linked. Multipoint parametric analysis estimated the position of $\mathrm{Ho}$ to a $\sim 7.4 \mathrm{cM}$ region (1-LOD decrease) flanked by OarSEJ12 and AGLA226, with a maximum $L O D=8.78$ at position $41.5 \mathrm{cM}$ (Figure 2). This area corresponds to the region between 25 and $31.6 \mathrm{Mb}$ on cattle chromosome 12 (Btau release 4.0 ) which contains $\sim 52$ genes.

\section{Horn morphology: variance component estimation and QTL mapping}

Variance component analyses provided estimates of the magnitude of various random effects, under the null hypothesis of no segregating QTL (Table 2).

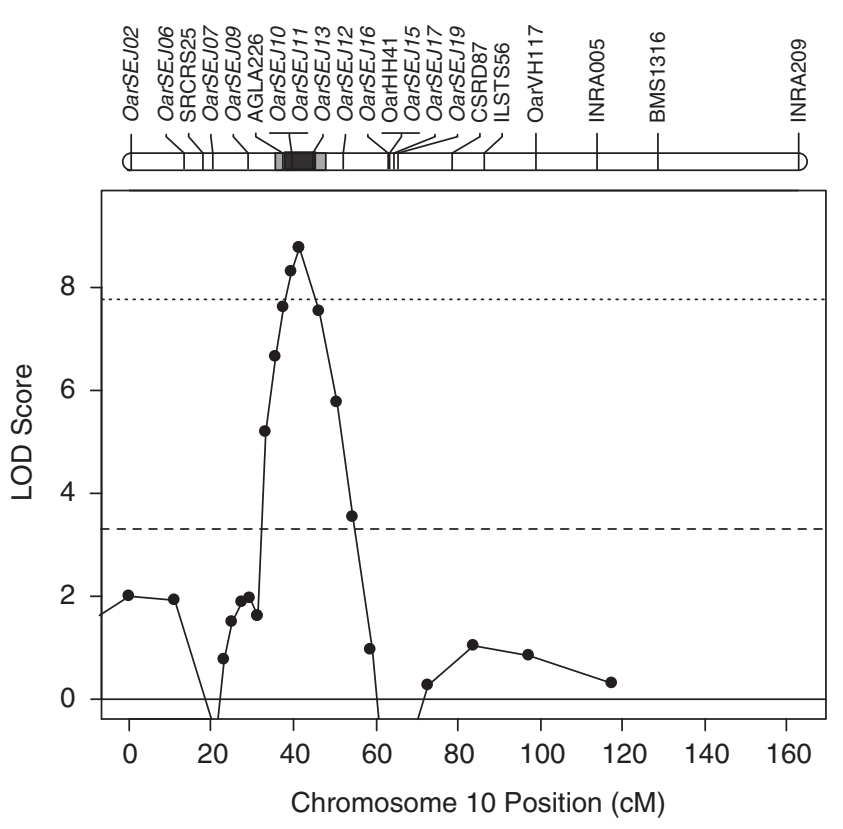

Figure 2 Parametric multi-point mapping of Ho. Horn type was tested simultaneously with four markers (SRCRS25, OarSEJ09, OarSEJ10 and OarSEJ16) and the putative Ho locus. The chromosome 10 linkage map is shown along the top axis, with the estimated position of $\mathrm{Ho}$ represented by dark grey and light grey shading for the 95 and $99 \%$ confidence intervals, respectively; the map was drawn using MapChart 2.1 (Voorrips, 2002). The position of $\mathrm{Ho}$ was tested at intervals corresponding to recombination fractions of 0.05 from each marker as specified in LINKAGE. The dashed line denotes the theoretical genome-wide significance threshold; the dotted lines denote the 1-LOD decrease and 95\% confidence interval from the peak LOD of 8.78 at $41.5 \mathrm{cM}$.

Horn length: This trait had a significant heritable component $\left(h^{2}=0.327, \quad P<0.01\right)$ and permanent environmental effect $\left(c^{2}=0.250, \quad P<0.001\right)$ in the polygenic model. Birth year and capture year components were also significant $(P<0.001$; Table 2$)$. Suggestive evidence of a QTL for horn length was detected on chromosome 10 at $37 \mathrm{cM}$ (Figure 3: $\mathrm{LOD}=2.51$; $\mathrm{LRT}=12.02, P<0.001$ ), which corresponds to the position of $H o$. When the QTL effect was fitted, the additive genetic variance $\left(V_{\mathrm{A}}\right)$ and therefore the heritability collapsed to a value close to 0 , and the QTL effect was estimated as $q^{2}=0.334$. As it is unlikely that the QTL explains all of $V_{\mathrm{A}}$, we repeated the model removing constraints upon the heritability to fall within the theoretical parameter space, which resulted in non-zero estimate of the s.e. of $V_{\mathrm{A}}$, and consequently, the heritability $\left(h^{2}=-0.109\right.$, s.e. $\left.=0.125\right)$. Although it is impossible to have negative heritability, the s.e. indicates that $V_{\mathrm{A}}$ becomes very small when the QTL is fitted; therefore, the QTL explains a large proportion of $V_{\mathrm{A}}$ in this trait. The QTL confidence interval derived from the 1-LOD decrease support interval spanned $\sim 34 \mathrm{cM}$ (flanked by CSRD87 and OarSEJ09; Figure 3). A whole genome scan provided no evidence of suggestive QTL for horn length elsewhere in the genome.

Horn circumference: This trait had a significant heritable component $\left(h^{2}=0.442, \quad P<0.001\right)$ in the polygenic model. Birth year and capture year 


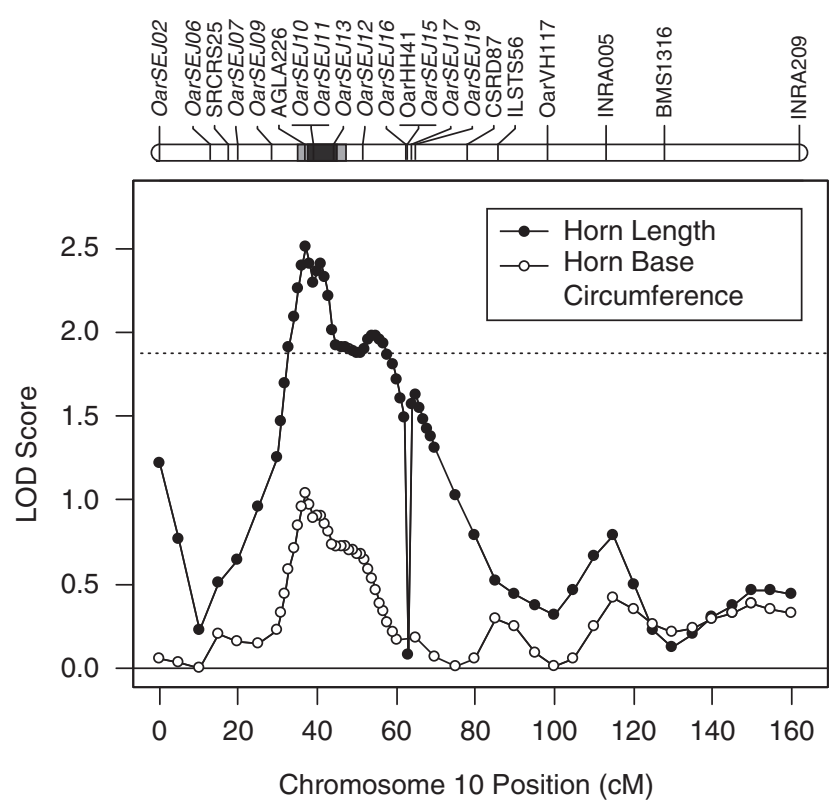

Figure 3 Map positions of putative quantitative trait loci (QTL) for total horn length (black circles) and horn base circumference (white circles) in normal-horned males. LOD score represents the probability of having a QTL in a given position against the probability of no QTL at that position. The horizontal dotted line represents the threshold for a suggestive QTL $(\mathrm{LOD}=1.88)$. The top axis shows marker positions on chromosome 10, with the estimated position of Ho represented by dark grey and light grey shading for the 95 and $99 \%$ confidence intervals, respectively.

components were also significant $(P<0.036$ and $P<0.004$, respectively; Table 2) There was evidence of a QTL at $37 \mathrm{cM}$, corresponding to $\mathrm{Ho}$, which fell below the suggestive threshold but was still significant using an LRT (Figure 3: $\mathrm{LOD}=1.04$, $\mathrm{LRT}=4.78, P=0.014$ ). The QTL effect was estimated as $q^{2}=0.237$, and explained a large proportion of $V_{\mathrm{A}}$ as the heritability decreased to $h^{2}=0.124$ (Table 2). As the LOD score was marginally $>1$, an accurate confidence interval for any QTL effect could not be inferred.

Bivariate analysis of horn length and base circumference In the bivariate polygenic model, there was a strong phenotypic and genetic correlation between horn length and base circumference $\left(r_{\mathrm{P}}=0.754\right.$, s.e. $=0.026$ and $r_{\mathrm{A}}=0.730$, s.e. $\left.=0.099\right)$. Fitting a bivariate QTL effect at position $37 \mathrm{cM}$, the most likely position of both horn length and base circumference QTLs, significantly improved the model compared with the polygenic model $(\mathrm{LRT}=11.7$, d.f. $=3, P<0.008)$. Similar to the univariate models, fitting the QTL effect resulted in the polygenic genetic correlation collapsing to zero. There was a significant positive phenotypic correlation $\left(r_{\mathrm{P}}=0.752\right.$, s.e. $=0.027)$ and a positive QTL effect correlation $\left(r_{\mathrm{Q}}=0.983\right.$, s.e. $\left.=0.081\right)$ between the traits. The full model was also a significant improvement on three additional models, the first two fitting the QTL effect on either length (full model vs. length QTL only, LRT $=9.08$, d.f. $=2, P=0.011$ ) or base circumference only (full model vs. circumference QTL only, $\mathrm{LRT}=10.72$, d.f. $=2$, $P=0.005)$, and the third constraining the covariance at 
the QTL to zero (full model vs. no covariance between length and circumference QTL, LRT $=8.9$, d.f. $=1$, $P=0.003)$. However, the full model was not a better fit than was the model in which the QTL correlation was constrained to one (LRT $=0.02$, d.f. $=2, P>0.999$ ). In other words, the genetic correlation between horn length and horn circumference is determined by a QTL located in the same genomic region as $H o$, which affects both traits in the same direction (that is, QTL alleles for long horns also confer horns with a large base circumference).

\section{Discussion}

We mapped Ho in the Hirta Soay sheep population to a region where marker tests may soon be developed, enabling the discrimination between animals with similar phenotype, but with a different underlying genotype. We also showed that both horn type in all the Soay sheep and horn length and base circumference in normal-horned male Soay sheep are likely to be controlled by the same chromosomal region. This is indicated by the co-localization of a QTL for horn morphology traits with $H o$ on chromosome 10. Our results provide a foundation for examining selection on horn type and morphology at the level of genotype, and will improve our understanding of how the horns polymorphism is maintained in this population.

\section{Mapping $\mathrm{Ho}$ and horn morphology QTL}

We located $H o$ in the same region of sheep chromosome 10 described in the studies by Beraldi et al. (2006) and Montgomery et al. (1996), and reduced the confidence interval from $\sim 16$ to $7.4 \mathrm{cM}$ in this population. The LOD score increased from 5.10 to 8.78 , and an association with four additional markers strengthens the evidence that $H o$ in Soay sheep lies within the confidence interval described above. Furthermore, we located a suggestive QTL spanning the Ho confidence interval, which may account for the majority of heritable variation in horn length and base circumference in normal-horned males. A genome scan provided no other suggestive QTL for horn length or base circumference; combined with an $a$ priori knowledge of the position of $H o$, it is likely that the QTLs at this position are not due to type 1 error or effect overestimation. The QTL model for horn base circumference at $37 \mathrm{cM}$ was nominally significant using a LRT, but the LOD score was below the threshold for suggestive linkage. However, given our knowledge of the position and effects of $H o$ and the horn length QTL, as well as a strong positive phenotypic correlation between length and circumference, our data suggest that $H o$ is responsible for a significant proportion of quantitative variation in base circumference. Furthermore, $\mathrm{Ho}$ appears to explain the variation in horn size, independently of any effect that overall body size QTL might have on horns (see Supplementary information).

\section{Estimation of QTL effect and QTL significance}

Estimates of QTL effect in free-living populations may often be upwardly biased, as data sets with relatively low sample size and power typically focus attention on the overestimated QTL because of what is known as the 'Beavis effect' (Beavis, 1998). However, we are confident that the QTL estimates are not dramatically biased for several reasons. First, it has been shown under a range of pedigree structures that the two-step variance components measure does not usually result in the large upward bias of QTL effects and downward bias of remaining polygenic effects (George et al., 2000). Second, our analysis focused on a specific region a priori, and hence is less prone to errors in QTL magnitude estimation, which are most severe when a whole genome scan is conducted and the largest QTL reported without previous knowledge of their likely location. Third, we attempted to reduce the upward bias when estimating additive genetic and/or QTL effects by fitting permanent environmental effects and common environmental effects (such as birth year and capture year) as random effects rather than as fixed effects. Fitting them as fixed effects would mean that they did not contribute to the overall non-genetic variance $\left(V_{\mathrm{P}}\right)$ and therefore, result in higher estimates of $q^{2}$ and $h^{2}$. For a similar discussion of how fitting of fixed effects can result in upwardly biased estimates of heritability, see the studies by Wilson (2008) and Visscher et al. (2008). In time, it may be possible to empirically test whether estimates of QTL magnitude were biased by re-estimating them on separate, unrelated sibships. The strong positive correlation between the QTLs for horn length and horn circumference indicates that gene(s) in this region, which confer longer horns, will also confer horns with a larger horn circumference. Our results provide compelling evidence that a small number of genes of large effect explain much of the genetic variation in a quantitative trait under selection in the wild.

\section{Bivariate QTL analysis}

As far as we are aware, this is the first time that a bivariate animal model has been used to explore the nature of genetic correlations at a QTL in the wild. Univariate analyses are capable of showing that two traits share co-localized QTL, but not whether correlations of QTL allelic effects are positive or negative (or zero, if the alleles at the two QTLs are not in linkage disequilibrium). By estimating the magnitude and direction of QTL correlations, it is possible to gain additional insight into the shared genetic architecture of traits and to predict the evolutionary consequences of selection on a given trait. A similar approach could be adopted to explore QTL by environmental interactions, for example, by treating a trait measured in one environment as trait one and that measured in a second environment as trait two.

\section{Selection on the Ho genotype}

Most analyses of selection and evolution of horns in the Soay sheep have focused either on horn type (among all animals; Clutton-Brock et al., 1997; Robinson et al., 2006) or on horn length (among normal-horned males; Preston et al., 2003; Robinson et al., 2008). In this study, we show that the two types of trait have a correlated genetic basis, and hence the evolution of horn type is likely to impact on horn length (and vice versa). Therefore, the evolution of the Ho genotype may be driven by more factors than previously believed. It is particularly interesting that the polled allele $\left(\mathrm{Ho}^{\mathrm{P}}\right)$ has persisted in the population despite polled females $\left(\mathrm{Ho}^{\mathrm{P}} \mathrm{Ho}^{\mathrm{P}}\right.$ or $\left.H o^{\mathrm{L}} H o^{\mathrm{P}}\right)$ and scurred males $\left(H o^{\mathrm{P}} H o^{\mathrm{P}}\right)$ being the least fit phenotypes in both sexes (Robinson et al., 2006). One possibility is that genotypes 
including the polled allele in non-polled sheep (such as $\mathrm{Ho}^{+} \mathrm{Ho}^{\mathrm{P}}$ in scurred females and $\mathrm{Ho}^{+} \mathrm{Ho}^{\mathrm{P}}$ and $\mathrm{Ho}^{\mathrm{L}} \mathrm{Ho} \mathrm{o}^{\mathrm{P}}$ in normal-horned males) are fitter than other genotypes conferring similar phenotypes, and are maintained in the population by balancing selection. Differences in fitness between sheep with similar phenotypes, but with different underlying genotypes, may also occur through associations with other alleles at adjacent loci in linkage disequilibrium with $H o$. It is only by identifying and typing the Ho locus that we will be able to investigate these hypotheses completely.

\section{Future directions}

Future investigations of horns evolution in the Hirta Soay sheep population will benefit from investigating the relationship between the $H o$ genotype and fitness. We aim to develop a genetic test for the Ho genotype in the Soay sheep, by identifying a marker in linkage disequilibrium with $H o$ or by identifying the causal mutations responsible for differences in horn type. Resources to further dissect the genetic basis of horns variation are becoming more readily available (http://www.sheephapmap.org/), facilitating the next phase in gene discovery. Identifying different haplotypes corresponding to the three $\mathrm{Ho}$ alleles and comparing the levels of variation on each haplotype should allow us to infer which horn types are ancestral and when different alleles arose within the population.

Given that the Soay sheep are a unique island population, replication of the results in this paper poses an additional challenge. Genetic variation in horn length and morphology has also been the focus of evolutionary genetics research in the bighorn sheep, Ovis canadensis (Coltman et al., 2005; Poissant et al., 2008), and extensive DNA and phenotype information for domestic sheep is becoming available for further study. Therefore, a possible extension is to examine whether the same chromosomal region also explains a significant proportion of the additive genetic variation in horn length in these other populations, to establish whether the same set of genes explain variation in different breeds and species. Potentially, horn type and morphology in sheep are an ideal system in which to investigate whether the same genes are important in macroevolutionary and microevolutionary processes in nature.

\section{Acknowledgements}

We thank Jill Pilkington, Soay sheep project members and hundreds of volunteers who have collected morphological data and tissue samples during the course of the long-term study on St Kilda. We also thank Alastair Wilson, Jake Gratten, Peter Visscher, Andy Krupa, Gavin Horsburgh and Nathan Haigh for their valuable discussions and guidance regarding many aspects of data analysis and laboratory work. Comments from the editor and three anonymous reviewers helped to improve this paper. The permission to work on St Kilda was granted by the National Trust for Scotland, and logistical support was provided by QinetiQ. A NERC thematic program 'Environmental Genomics' funded the development of the Soay sheep mapping pedigree and the genotyping of markers throughout the Soay sheep genome. SEJ is supported by a BBSRC CASE Studentship with Catapult Genetics.

\section{References}

Almasy L, Blangero J (1998). Multipoint quantitative-trait linkage analysis in general pedigrees. Am J Hum Genet 62: 1198-1211.

Altschul SF, Gish W, Miller W, Myers EW, Lipman DJ (1990). Basic local alignment search tool. J Mol Biol 215: 403-410.

Andersson M (1994). Sexual Selection. Princeton University Press: Princeton, NJ, USA.

Beavis WD (1998). QTL analyses: power, precision, and accuracy. In: Paterson AH (ed). Molecular Dissection of Complex Traits. CRC Press: New York. pp 145-162.

Beraldi D, McRae AF, Gratten J, Pilkington JG, Slate J, Visscher PM et al. (2007a). Quantitative trait loci (QTL) mapping of resistance to strongyles and coccidia in the free-living Soay sheep (Ovis aries). Int J Parasitol 37: 121.

Beraldi D, McRae AF, Gratten J, Slate J, Visscher PM, Pemberton JM (2006). Development of a linkage map and mapping of phenotypic polymorphisms in a free-living population of Soay sheep (Ovis aries). Genetics 173: 1521-1537.

Beraldi D, McRae AF, Gratten J, Slate J, Visscher PM, Pemberton JM (2007b). Mapping quantitative trait loci underlying fitness-related traits in a free-living sheep population. Evolution 61: 1403-1416.

Brownstein MJ, Carpten JD, Smith JR (1996). Modulation of non-templated nucleotide addition by Taq DNA polymerase: primer modifications that facilitate genotyping. Biotechniques 20: 1004-1010.

Charmantier A, Perrins C, McCleery R, Sheldon BC (2006). Agedependent genetic variance in a life-history trait in the mute swan. Proc R Soc Lond B 273: 225-232.

Clutton-Brock TH, Pemberton JM (2004a). Individuals and populations. In: Clutton-Brock TH and Pemberton JM (eds). Soay Sheep: Dynamics and Selection in an Island Population. Cambridge University Press: Cambridge, UK. pp 1-16.

Clutton-Brock TH, Pemberton JM (2004b). Soay Sheep: Dynamics and Selection in an Island Population. Cambridge Univ Press: Cambridge, UK.

Clutton-Brock TH, Pemberton JM, Coulson T, Stevenson IR, MacColl ADC (2004). The sheep of St Kilda. In: CluttonBrock TH and Pemberton JM (eds). Soay Sheep: Dynamics and Selection in an Island Population. Cambridge University Press: Cambridge, UK.

Clutton-Brock TH, Wilson K, Stevenson IR (1997). Densitydependent selection on horn phenotype in Soay sheep. Philos Trans $R$ Soc B 352: 839-850.

Coltman DW, Bancroft DR, Robertson A, Smith JA, CluttonBrock TH, Pemberton JM (1999). Male reproductive success in a promiscuous mammal: behavioural estimates compared with genetic paternity. Mol Ecol 8: 1199-1209.

Coltman DW, O'Donoghue P, Hogg JT, Festa-Bianchet M (2005). Selection and genetic (co)variance in bighorn sheep. Evolution 59: 1372-1382.

Coltman DW, Pemberton JM (2004). Inheritance of coat colour and horn type in Soay sheep. In: Clutton-Brock TH and Pemberton JM (eds). Soay Sheep: Dynamics and Selection in an Island Population. Cambridge University Press: Cambridge, UK. pp 321-327.

Dolling CHS (1961). Hornedness and polledness in sheep. IV. Triple alleles affecting horn development in the Merino. Aust J Agric Res 12: 353-361.

Ellegren H, Sheldon BC (2008). Genetic basis of fitness differences in natural populations. Nature 452: 169-175.

Falconer DS, Mackay TFC (1996). Introduction to Quantitative Genetics 4th edn Longman: UK.

Fisher RA (1958). The Genetics Theory of Natural Selection. Dover: New York, USA. 
Garant D, Kruuk LEB, Wilkin TA, McCleery RH, Sheldon BC (2005). Evolution driven by differential dispersal within a wild bird population. Nature 433: 60-65.

George AW, Visscher PM, Haley CS (2000). Mapping quantitative trait loci in complex pedigrees: a two-step variance component approach. Genetics 156: 2081-2092.

Gilmour AR, Gogel BJ, Cullis BR, Welham SJ, Thompson R (2002). ASReml User Guide Release 1.0. VSN International Ltd: Hemel Hempstead, UK.

Gratten J, Beraldi D, Lowder BV, McRae AF, Visscher PM, Pemberton JM et al. (2007). Compelling evidence that a single nucleotide substitution in TYRP1 is responsible for a coatcolour polymorphism in a free-living population of Soay sheep. Proc $R$ Soc Lond B 274: 619-626.

Gratten J, Wilson AJ, McRae AF, Beraldi D, Visscher PM, Pemberton JM et al. (2008). A localized negative genetic correlation constrains microevolution of coat colour in wild sheep. Science 319: 318-320.

Green P, Falls K, Crooks S (1990). Documentation for CRIMAP, version 2.4. Washington University School of Medicine: St Louis, MO, USA.

Heath SC (1997). Markov chain Monte Carlo segregation and linkage analysis for oligogenic models. Am J Hum Genet 61: 748-760.

Houle D (1992). Comparing evolvability and variability of quantitative traits. Genetics 130: 195-204.

Jensen H, Steinsland I, Ringsby TH, Sæther B-E (2008). Evolutionary dynamics of a sexual ornament in the house sparrow (Passer domesticus): the role of indirect selection within and between sexes. Evolution 62: 1275-1293.

Kalinowski ST, Taper ML, Marshall TC (2007). Revising how the computer program Cervus accommodates genotyping error increases success in paternity assignment. Mol Ecol 16: 1099-1106.

Kruuk LEB (2004). Estimating genetic parameters in natural populations using the 'animal model'. Philos Trans $R$ Soc $B$ 359: 873-890.

Kruuk LEB, Clutton-Brock TH, Slate J, Pemberton JM, Brotherstone S, Guinness FE (2000). Heritability of fitness in a wild mammal population. PNAS 97: 698-703.

Kruuk LEB, Slate J, Pemberton JM, Brotherstone S, Guinness FE, Clutton-Brock TH (2002). Antler size in red deer: heritability and selection but no evolution. Evolution 56: 1683-1695.

Kruuk LEB, Slate J, Wilson AJ (2008). New answers for old questions: the evolutionary quantitative genetics of wild animal populations. Annu Rev Ecol Evol Syst 39: 525-548.

Lander ES, Botstein D (1989). Mapping Mendelian factors underlying quantitative traits using RFLP linkage maps. Genetics 121: 185-199.

Lander ES, Kruglyak L (1995). Genetic dissection of complex traits: guidelines for interpreting and reporting linkage results. Nat Genet 11: 241-247.

Liu X, Grosz M (2006). Animal Genomics and Breed Group. Monsanto Company: St Louis, MO, USA.

Lynch M, Walsh B (1998). Genetics and Analysis of Quantitative Traits. Sinauer Associates Inc.: Sunderland, MA, USA.

Merilä J, Sheldon BC (1999). Genetic architecture of fitness and nonfitness traits: empirical patterns and development of ideas. Heredity 83: 103-109.

Montgomery GW, Henry HM, Dodds KG, Beattie AE, Wuliji T, Crawford AM (1996). Mapping the horns $(\mathrm{Ho})$ locus in sheep: a further locus controlling horn development in domestic animals. J Hered 87: 358-363.

Nussey DH, Postma E, Gienapp P, Visser ME (2005). Selection on heritable phenotypic plasticity in a wild bird population. Science 310: 304-306.
Nyholt DR (2000). All LODs are not created equal. Am J Hum Genet 67: 282-288.

O'Connell JR, Weeks DE (1998). PedCheck: a program for identification of genotype incompatibilities in linkage analysis. Am J Hum Genet 63: 259-266.

Overall ADJ, Byrne KA, Pilkington JG, Pemberton JM (2005). Heterozygosity, inbreeding and neonatal traits in Soay sheep on St Kilda. Mol Ecol 14: 3383-3393.

Poissant J, Wilson AJ, Festa-Bianchet M, Hogg JT, Coltman DW (2008). Quantitative genetics and sex-specific selection on sexually dimorphic traits in bighorn sheep. Proc $R$ Soc Lond B 275: 623-628.

Pomiankowski A, Møller AP (1995). A resolution of the lek paradox. Proc R Soc Lond B 260: 21-29.

Preston BT, Stevenson IR, Pemberton JM, Coltman DW Wilson K (2003). Overt and covert competition in a promiscuous mammal: the importance of weaponry and testes size to male reproductive success. Proc $R$ Soc Lond $B$ 270: 633-640.

R Development Core Team (2008). R: a language and environment for statistical computing. Available at: http://www. R-project.org.

Réale D, Berteaux D, McAdam AG, Boutin S (2003). Lifetime selection on heritable life-history traits in a natural population of red squirrels. Evolution 57: 2416-2423.

Robinson MR, Pilkington JG, Clutton-Brock TH, Pemberton JM, Kruuk LEB (2006). Live fast, die young: trade-offs between fitness components and sexually antagonistic selection on weaponry in Soay sheep. Evolution 60: 2168-2181.

Robinson MR, Pilkington JG, Clutton-Brock TH, Pemberton JM, Kruuk LEB (2008). Environmental heterogeneity generates fluctuating selection on a secondary sexual trait. Curr Biol 18 : 751-757.

Rozen S, Skaletsky HJ (2000). PRIMER3 on WWW for general users and for biologist programmers. In: Krawets $S$ and Misener S (eds). Bioinformatics Methods and Protocols: Methods in Molecular Ecology. Humana Press: Totowa, NJ, USA. pp 365-396.

Slate J (2005). Quantitative trait locus mapping in natural populations: progress, caveats and future directions. Mol Ecol 14: 363-379.

Slate J, Visscher PM, MacGregor S, Stevens DR, Tate ML, Pemberton JM (2002). A genome scan for quantitative trait loci in a wild population of red deer (Cervus elaphus). Genetics 162: 1863-1873.

Terwilliger JD, Ott J (1994). Handbook of Human Genetic Linkage. John Hopkins University Press: Baltimore, MD, USA.

Thériault V, Garant D, Bernatchez L, Dodson JJ (2007). Heritability of life-history tactics and genetic correlation with body size in a natural population of brook charr (Salvelinus fontinalis). J Evol Biol 20: 2266-2277.

Visscher PM, Hill WG, Wray NR (2008). Heritability in the genomics era - concepts and misconceptions. Nature Rev Genet 9: 255-266.

Voorrips RE (2002). MapChart: software for the graphical presentation of linkage maps and QTLs. J Hered 93: 77-78.

Wigginton JE, Abecasis GR (2005). PEDSTATS: descriptive statistics, graphics and quality assessment for gene mapping data. Bioinformatics 21: 3445-3447.

Wilson AJ (2008). Why $h^{2}$ does not always equal $\mathrm{V}_{\mathrm{A}} / \mathrm{V}_{\mathrm{P}}$ ? J Evol Biol 21: 647-650.

Wilson AJ, Kruuk LEB, Coltman DW (2005). Ontogenetic patterns in heritable variation for body size: using random regression models in a wild ungulate population. Am Nat 166: E177-E192.

Supplementary Information accompanies the paper on Heredity website (http://www.nature.com/hdy) 